Supporting information

\title{
Linkage between Particulate Matter Properties and Lung Function in Schoolchildren: A Panel Study in Southern China
}

Qiansheng $\mathrm{Hu}^{1 \#}$, Xiaoyan $\mathrm{Ma}^{1 \#}$, Dingli Yue ${ }^{2}$, Jiajia Dai ${ }^{1}$, Lu Zhao ${ }^{1}$, Dan Zhang ${ }^{1}$, Deji

Ciren ${ }^{1}$, Jianqing Lin ${ }^{1}$, Boning You ${ }^{1}$, Yuhong Zhai ${ }^{2}$, Luan Yuan ${ }^{2}$, Weiwei Lin ${ }^{1 *}$

${ }^{1}$ Department of Occupational and Environmental Health, School of Public Health, Sun Yat-Sen University, Guangzhou, 510080, China

${ }^{2}$ Guangdong Environmental Monitoring Center, State Environmental Protection Key Laboratory of Regional Air Quality Monitoring, Guangzhou, 510308, China

${ }^{\#}$ Co-first authors. These authors contributed equally to this work.

${ }^{*}$ Corresponding author

Address: Department of Occupational and Environmental Health, School of Public Health, Sun Yat-Sen University, Yuexiu District, Guangzhou 510080, China; Tel: +86 20 87334995; Email: linweiw5@mail.sysu.edu.cn

PAGES: 12

SUPPLEMENTAL TABLES: 2

SUPPLEMENTAL FIGURES: 8 


\section{Materials and methods}

\section{Lung function measurements}

Table S1. The number of observations for 57 children's lung function during the study period

\begin{tabular}{cccccccccc}
\hline & Mean & SD & Min & P5 & P25 & Median & P75 & P95 & Max \\
\hline Number of observations (day) & 15 & 7 & 1 & 4 & 10 & 18 & 21 & 22 & 23 \\
\hline
\end{tabular}

\section{Ambient air pollutant measurements}

Measurements on properties of ambient air pollution were conducted at the Heshan Atmospheric Supersite over the study period. The mass concentration of hourly $\mathrm{PM}_{2.5}$ (tapered element oscillating microbalance, TEOM 1405, Thermo, USA), organic carbon (OC) and elemental carbon (EC) (Sunset-OCEC, Sunset Lab Inc, USA), nitrate $\left(\mathrm{NO}_{3}{ }^{-}\right)$and sulfate $\left(\mathrm{SO}_{4}{ }^{2-}\right)$ in $\mathrm{PM}_{2.5}$ (an online gases and aerosols collector ion chromatography analysis system, GAC-IC, Peking University, China), particle number size distributions (Scanning Mobility Particle Sizer, SMPS, TSI model SMPS3936L75, TSI Inc., St. Paul, MN, USA) and meteorological parameters including temperature and relative humidity (WXT520, Vaisala, Germany) were observed on-line respectively. Before data processing, all monitoring records were first screened to ensure data validation. That is, missing measurements due to planned (e.g. maintenance, upgrading, calibrations) or unforeseen (e.g. equipment failures, power outages) reasons, and collected data that was not representative of the ambient air conditions due to collection errors or equipment malfunctions were not included for data analysis. A 24-hour period with less than $1 / 3$ missing hours was considered 
valid. The hourly average data capture rates for all the air pollutants in our study were all above 85\% $\left(\mathrm{PM}_{2.5}\right.$ : 94.7\%; EC: $93.3 \%$; OC: $94.7 \%$; TC: $94.7 \%$; $\mathrm{SO}_{4}{ }^{2-}: 87 \%$; $\mathrm{NO}_{3}{ }^{-}$: 87\%; particle number concentration: 96\%).

The principle and configuration of the GAC-IC system were similar to that introduced in detail by Dong et al.. ${ }^{1}$ In the system, a set of dull-polished wet annular denuder with a steam jet aerosol collector was employed for the continuous sampling of both gas- and aerosol-phase pollutants, and the inorganic gaseous and particulate species were detected by two separate ion chromatography (IC; ICS-90, Dionex, USA). ${ }^{2}$

SMPS3936L75 consisted of a long differential mobility analyzer (TSI model 3081) and a Condensational Particle Counter (CPC, TSI model 3775) to monitor the particle number size distribution from 19.1 to $687.8 \mathrm{~nm}$. The system was kept dry with silica gel in the sample lines. Size-dependent diffusion losses within the inlet were corrected with empirical particle loss corrections, as given by Willeke and Baron. ${ }^{3}$ These particles were divided into nucleation mode $(<20 \mathrm{~nm})$, Aitken mode $(20-100 \mathrm{~nm})$ and accumulation mode (100-697.8 $\mathrm{nm}$ ) according to their diameters. The nucleation mode was not discussed because its initial observation segment was $19.1 \mathrm{~nm}$ in the study, close to the upper limit size of $20 \mathrm{~nm}$.

\section{Statistical Analysis}

\section{Random forest (RF) regression}

Random forest (RF) is a supervised learning algorithm that contains a set of tree-structured classifiers on various subsets of the given dataset, and each tree casts a 
unit vote for the most popular class. ${ }^{4}$ It is a powerful classification and regression approach for measuring the importance of variables and examining the extent to which each variable contributes to the effect estimate as a part of the results. ${ }^{4}$ Instead of relying on a single learning model, RF algorithm uses multiple decision models to increase predictive accuracy. The primary difference between RF and the standard decision tree algorithms is that variables used to evaluate at each tree node are generated randomly. And compared to other dimension reduction methods such as principal component analysis, discriminant analysis, support vector machines and neural networks, RF does better in preventing overfitting. ${ }^{4,5}$ Due to higher accuracy and robustness against overfitting, and the capability of providing insights about the behavior of the variable importance, RF has gained considerable popularity in the field of bioinformatics. ${ }^{6,7}$

The algorithm operates by extracting several random subsets, forming bootstrap sample of the observations, generating a large number of decision trees $(\mathrm{N})$, and letting these classifiers "vote" to form the final predictor. ${ }^{4}$ The variable importance measures in the regression are dependent on two indicators: "Increase in MSE (mean square error)" and "Increase in Node Purity"; the latter measures the loss function when best tree nodes are selected, while "Increase in MSE" offers the prediction ability of MSE with randomly permuted variable; the larger the value of both indicators is, the more important the variable is. ${ }^{4,8}$

$\mathrm{N}$ (the number of trees) and $\mathrm{M}$ (the number of variables in the subset at each node) are two important parameters in RF regression. To obtain importance measure for PM 
metrics, first, several bootstrap samples $(\mathrm{N})$ were randomly drawn from the original data as the training sets for growing the trees. For each of the bootstrap samples, $\mathrm{m}$ $(\mathrm{m}<\mathrm{M}, \mathrm{m} \in \mathrm{M})$ number of the predictors was randomly selected out of $\mathrm{M}(\mathrm{M}=10$, representing 10 PM metrics which showed significant adverse effects on lung function in the GEE procedure) as split candidates. The out-of-bag (OOB) samples, approximately $1 / 3$ of the observations, were then used for prediction accuracy check. Finally, based on the percentage increase in MSE[\%IncMSE] and "Increase in Node Purity" [IncNodePurity], we estimated the variable importance and ranked the effects of the 10 PM exposure metrics on lung function. Calculation for the optimal parameter for $\mathrm{N}$ and $\mathrm{m}$ was performed using $\mathrm{N}=50$ to 1000 , and $\mathrm{m}=1$ to 10 to minimize mean of squared residuals of the RF algorithm (Figure $\mathrm{S} 1$ ). $\mathrm{N}=50$ and $\mathrm{m}=$ 9 were finally set to achieve optimal performance.

Sensitivity analyses in the RF regression including: i) excluding pollutants with high Pearson correlation coefficients that exceeded 0.7 (i.e. EC, TC, aitdn, aitds and aitdv) to eliminate the interference of collinearity; and ii) examine the effects of PM metrics in different lag patterns (lag1, lag 2 and lags 0-2). 


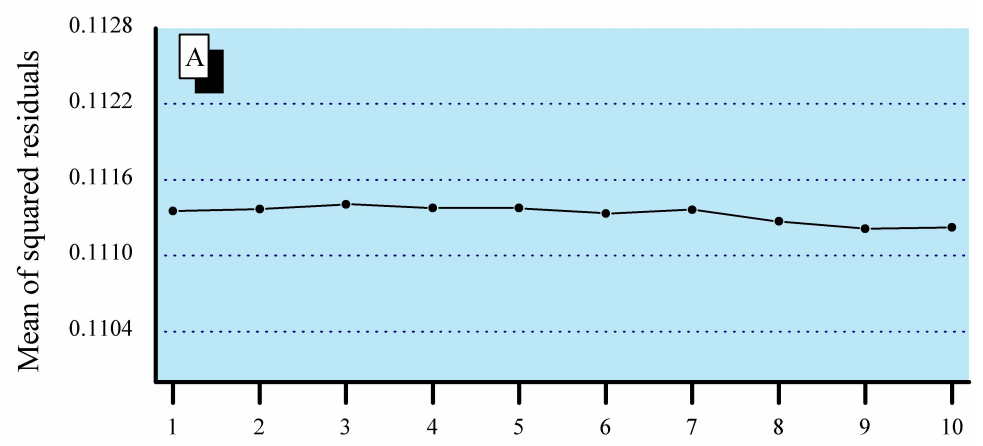

$\mathrm{m}$, the number of variables randomly sampled at each split

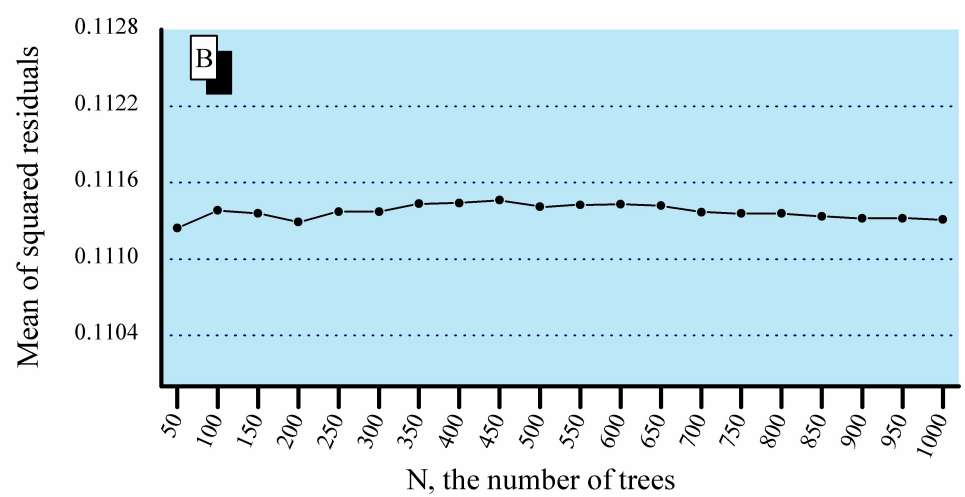

Figure S1. Mean of squared residuals under different $m$ (the number of variables randomly at each split, A) and $\mathrm{N}$ (the number of trees, B) when various PM metrics were incorporated into RF regression. 


\section{Results}
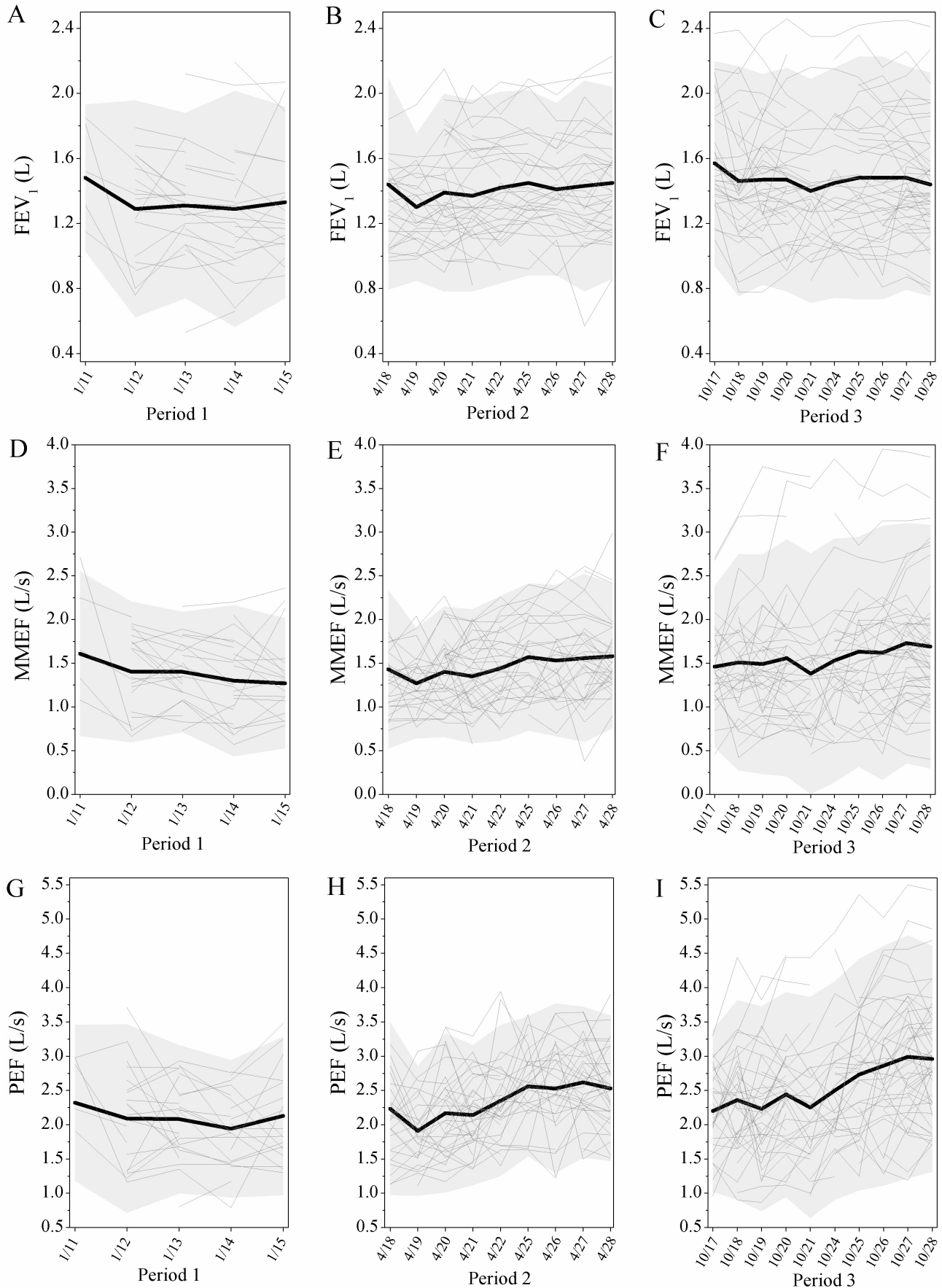

Figure S2. Day-to-day variations of lung function over all participating children during the three periods. The shaded area represents $95 \%$ CIs and each grey line represents daily variation of lung function in an individual subject. A, B, C: $\mathrm{FEV}_{1}$; D, E, F: MMEF; G, H, I: PEF. 

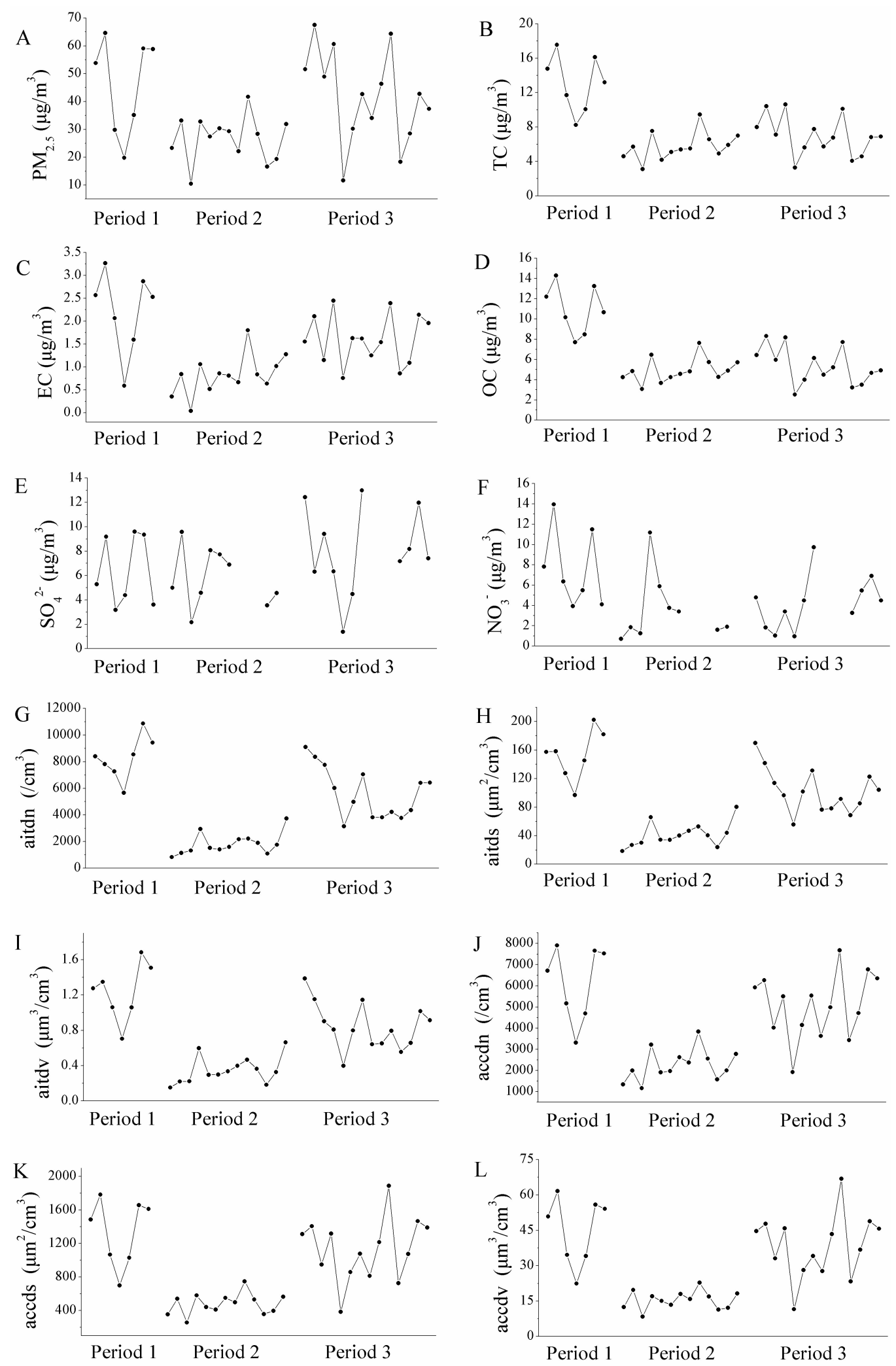

Figure S3. Temporal characteristics of air pollutant exposures. (A) $\mathrm{PM}_{2.5}$; (B) TC; (C) EC; (D) OC; (E) $\mathrm{SO}_{4}{ }^{2-}$; (F) $\mathrm{NO}_{3}{ }^{-}$; (G)Aitken mode particle number; (H) Aitken mode particle surface area; (I) Aitken mode particle volume; (J) accumulation mode particle number; (K) accumulation mode particle surface area and (L) accumulation mode particle volume. Period 1: 9-15 January; Period 2: 16-28 April; Period 3: 15-28 October. 
Table S2. The Pearson correlation coefficients of daily 24-hr mean concentrations of air pollution and weather correlation matrix of $\mathrm{PM}_{2.5}$ and its constituents and particle sizes.

\begin{tabular}{|c|c|c|c|c|c|c|c|c|c|c|c|c|c|c|}
\hline Variables & $\mathrm{PM}_{2.5}$ & $\mathrm{EC}$ & $\mathrm{OC}$ & $\mathrm{TC}$ & $\mathrm{SO}_{4}{ }^{2-}$ & $\mathrm{NO}_{3}{ }^{-}$ & Aitdn & Accdn & Aitds & Accds & Aitdv & Accdv & Temp & $\mathrm{RH}$ \\
\hline $\mathrm{PM}_{2.5}$ & 1.00 & - & - & - & - & - & - & - & - & - & - & - & - & - \\
\hline $\mathrm{EC}$ & $0.86^{* \#}$ & 1.00 & - & - & - & - & - & - & - & - & - & - & - & - \\
\hline $\mathrm{OC}$ & $0.68^{*}$ & $0.73^{* \#}$ & 1.00 & - & - & - & - & - & - & - & - & - & - & - \\
\hline $\mathrm{TC}$ & $0.76^{* \#}$ & $0.83^{* \#}$ & 0.99 *\# & 1.00 & - & - & - & - & - & - & - & - & - & - \\
\hline $\mathrm{SO}_{4}{ }^{2-}$ & $0.50 *$ & $0.32 *$ & $0.10^{*}$ & $0.17^{*}$ & 1.00 & - & - & - & - & - & - & - & - & - \\
\hline $\mathrm{NO}_{3}^{-}$ & $0.44 *$ & $0.48^{*}$ & $0.50^{*}$ & $0.52 *$ & $0.47 *$ & 1.00 & - & - & - & - & - & - & - & - \\
\hline Aitdn & $0.66^{*}$ & $0.78^{* \#}$ & $0.76^{* \#}$ & $0.80^{* \#}$ & $0.38 *$ & $0.43 *$ & 1.00 & - & - & - & - & - & - & - \\
\hline Accdn & $0.84 * \#$ & 0.93 *\# & $0.66^{*}$ & $0.76^{* \#}$ & $0.45^{*}$ & $0.53 *$ & 0.81 *\# & 1.00 & - & - & - & - & - & - \\
\hline Aitds & $0.68 *$ & $0.82 * \#$ & $0.79 * \#$ & $0.83^{* \#}$ & $0.37 *$ & $0.51 *$ & $0.98^{* \#}$ & $0.85^{* \#}$ & 1.00 & - & - & - & - & - \\
\hline Accds & $0.86^{* \#}$ & 0.91 *\# $^{\#}$ & $0.64 *$ & $0.74 *^{\#}$ & $0.46^{*}$ & $0.46^{*}$ & $0.78^{* \#}$ & $0.99 *^{\#}$ & $0.80 *^{\#}$ & 1.00 & - & - & - & - \\
\hline Aitdv & $0.73^{* \#}$ & $0.85^{* \#}$ & $0.79 *^{\#}$ & $0.85^{* \#}$ & $0.39 *$ & $0.56^{*}$ & $0.97 *^{\#}$ & $0.88^{* \#}$ & $0.99 * \#$ & $0.83 * \#$ & 1.00 & - & - & - \\
\hline Accdv & $0.87 *^{\#}$ & 0.90 *\# & $0.63^{*}$ & $0.73^{* \#}$ & $0.46^{*}$ & $0.43 *$ & $0.75^{* \#}$ & $0.98^{* \#}$ & 0.78 *\# & 1.00 *\# & $0.81{ }^{* \#}$ & 1.00 & - & - \\
\hline Temp & -0.09 & $-0.20 *$ & $-0.67 *$ & $-0.59 *$ & $0.24 *$ & $-0.25^{*}$ & $-0.47 *$ & $-0.20 *$ & $-0.50 *$ & $-0.15 *$ & $-0.45^{*}$ & $-0.14^{*}$ & 1.00 & - \\
\hline $\mathrm{RH}$ & $-0.39 *$ & $-0.34 *$ & $-0.35^{*}$ & $-0.37 *$ & $-0.53 *$ & $-0.12^{*}$ & $-0.57 *$ & $-0.37 *$ & $-0.50 *$ & $-0.38 *$ & $-0.48 *$ & $-0.38 *$ & -0.08 & 1.00 \\
\hline
\end{tabular}

${ }^{*} p<0.05$ from Pearson correlation test

${ }^{\#}$ Coefficients $>$ 0.7. Abbreviations: aitdn, Aitken mode particle number; accdn, accumulation mode particle number; aitds, Aitken mode particle surface area; accds, accumulation mode particle surface area; aitdv, Aitken mode particle volume; accdv, accumulation mode particle volume; Temp, temperature; RH, relative humidity. 


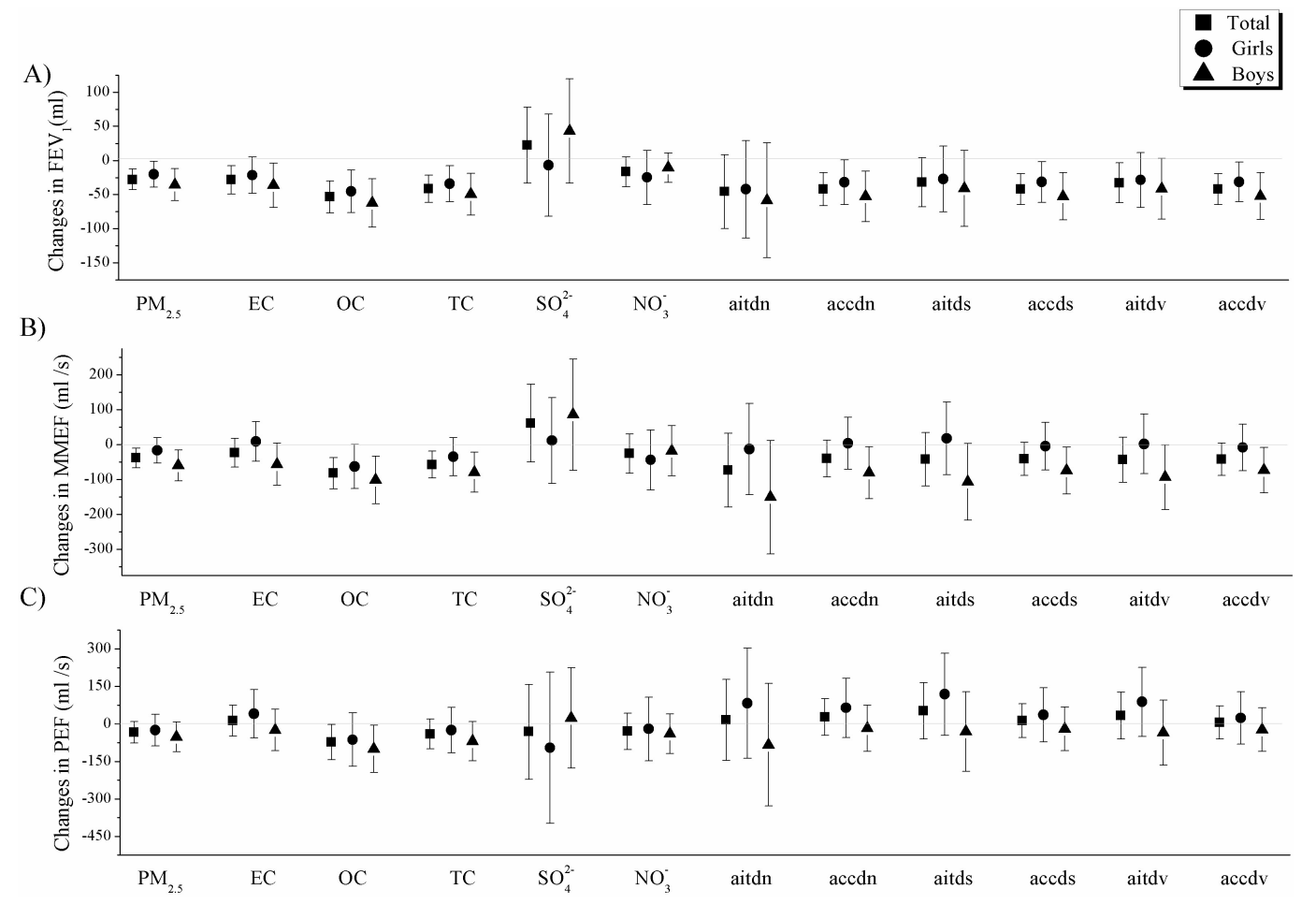

Figure S4. Changes in lung function parameters associated with an IQR increase in daily average (0-24 hr) concentrations of $\mathrm{PM}_{2.5}$ metrics stratified by gender in GEE models. Error bars are $95 \%$ CIs. A) $\mathrm{FEV}_{1}$; B) MMEF; C) PEF. All estimates are adjusted for age, BMI, ETS, temperature and $\mathrm{RH}$, day-of-week and period. The IQR concentrations for $\mathrm{PM}_{2.5}$ mass, $19.1 \mu \mathrm{g} / \mathrm{m}^{3}$; for EC, 1.2 $\mu \mathrm{g} / \mathrm{m}^{3}$; for $\mathrm{OC}, 3.6 \mu \mathrm{g} / \mathrm{m}^{3}$; for TC, $4.4 \mu \mathrm{g} / \mathrm{m}^{3}$; for $\mathrm{SO}_{4}{ }^{2-}, 3.8 \mu \mathrm{g} / \mathrm{m}^{3}$, for $\mathrm{NO}_{3}{ }^{-}, 2.6 \mu \mathrm{g} / \mathrm{m}^{3}$; for aitdn, $4902.3 / \mathrm{cm}^{3}$; for accdn, $3237.8 / \mathrm{m}^{3}$; for aitds, $76.2 \mu \mathrm{m}^{2} / \mathrm{cm}^{3}$; for accds, $710.5 \mu \mathrm{m}^{2} / \mathrm{cm}^{3}$; for aitdv, $0.6 \mu \mathrm{m}^{3} / \mathrm{cm}^{3}$ and for accdv, $25.2 \mu \mathrm{m}^{3} / \mathrm{cm}^{3}$. The abbreviations are the same as Table S2.

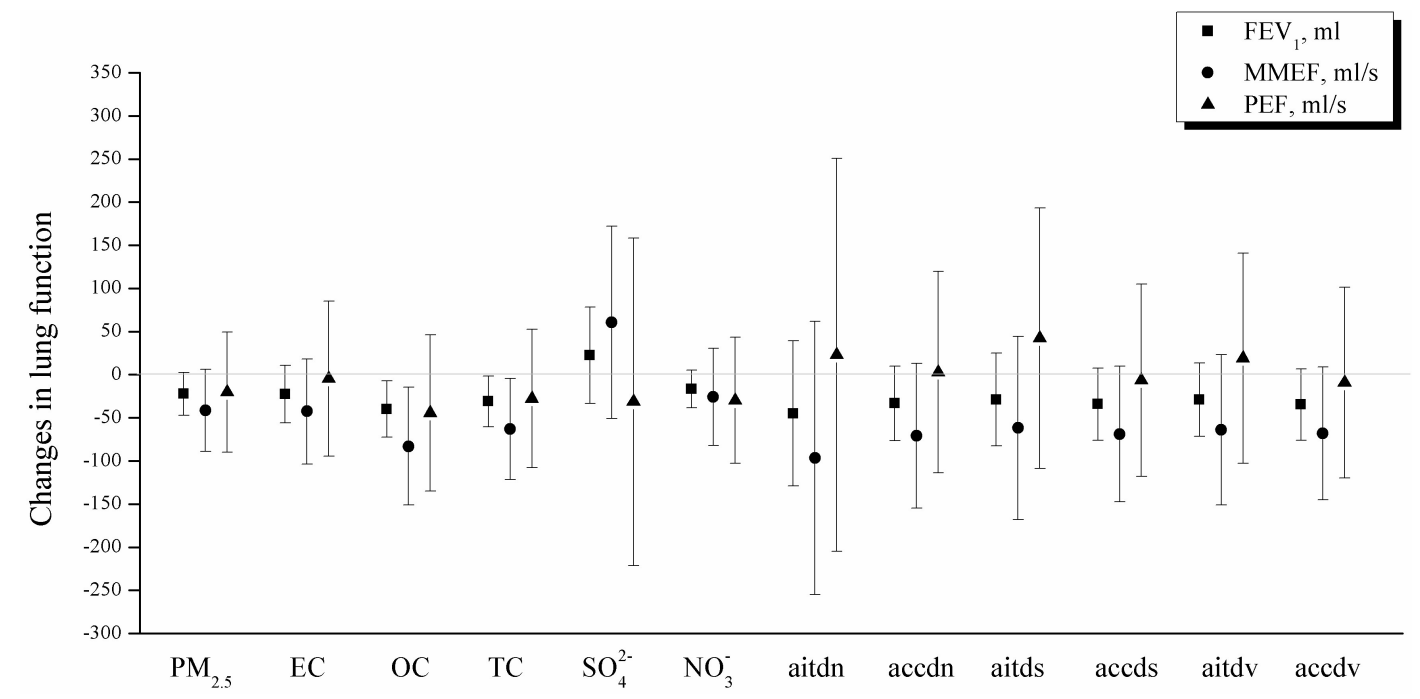

Figure S5. Changes with $95 \%$ CIs in lung function along with per IQR increase in $0-24 \mathrm{hr}$ concentrations of various $\mathrm{PM}_{2.5}$ metrics, which used five-day observation data for each study period, adjusted for age, gender, BMI, ETS, temperature, RH, day-of-week and period. The IQR concentrations for PM metrics and abbreviations are the same as Figure S4. 

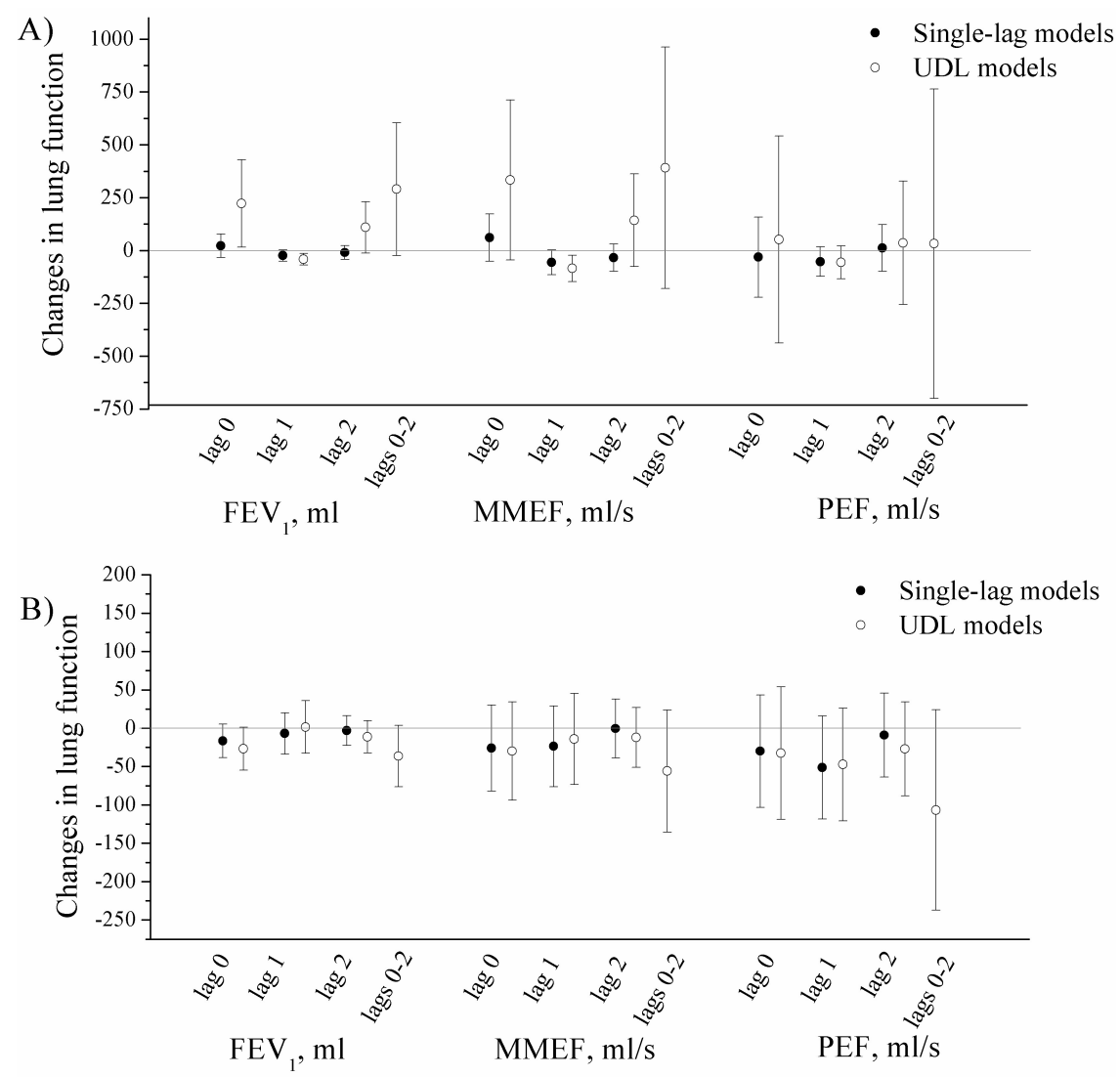

Figure S6. Estimated Changes with $95 \% \mathrm{CI}$ in lung function along with per IQR increase in $\mathrm{SO}_{4}{ }^{2-}$ and $\mathrm{NO}_{3}{ }^{-}$among different exposure lag times. A) $\mathrm{SO}_{4}{ }^{2-}$; B) $\mathrm{NO}_{3}{ }^{-}$. All models are adjusted for age, gender, BMI, ETS, temperature, $\mathrm{RH}$, day-of-week and period.
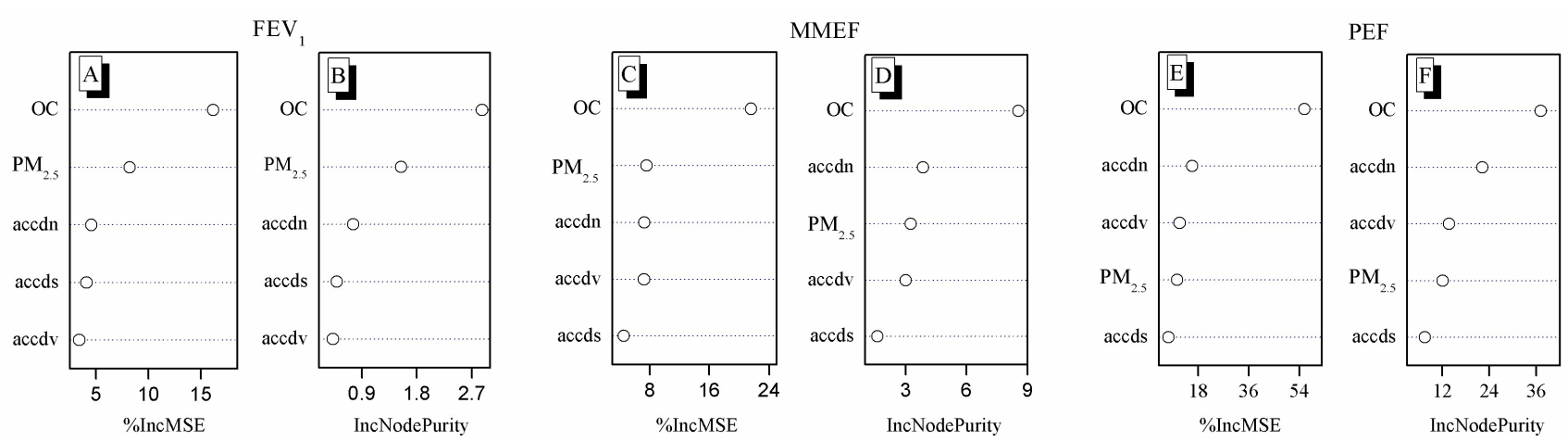

Figure S7. The relative importance of each $\mathrm{PM}_{2.5}$-metric on lung function after removal of pollutants with high correlation (TC, EC, aitdn, aitds, aitdv were excluded). A, B for $\mathrm{FEV}_{1}$; C, D for MMEF; E and F for PEF. 

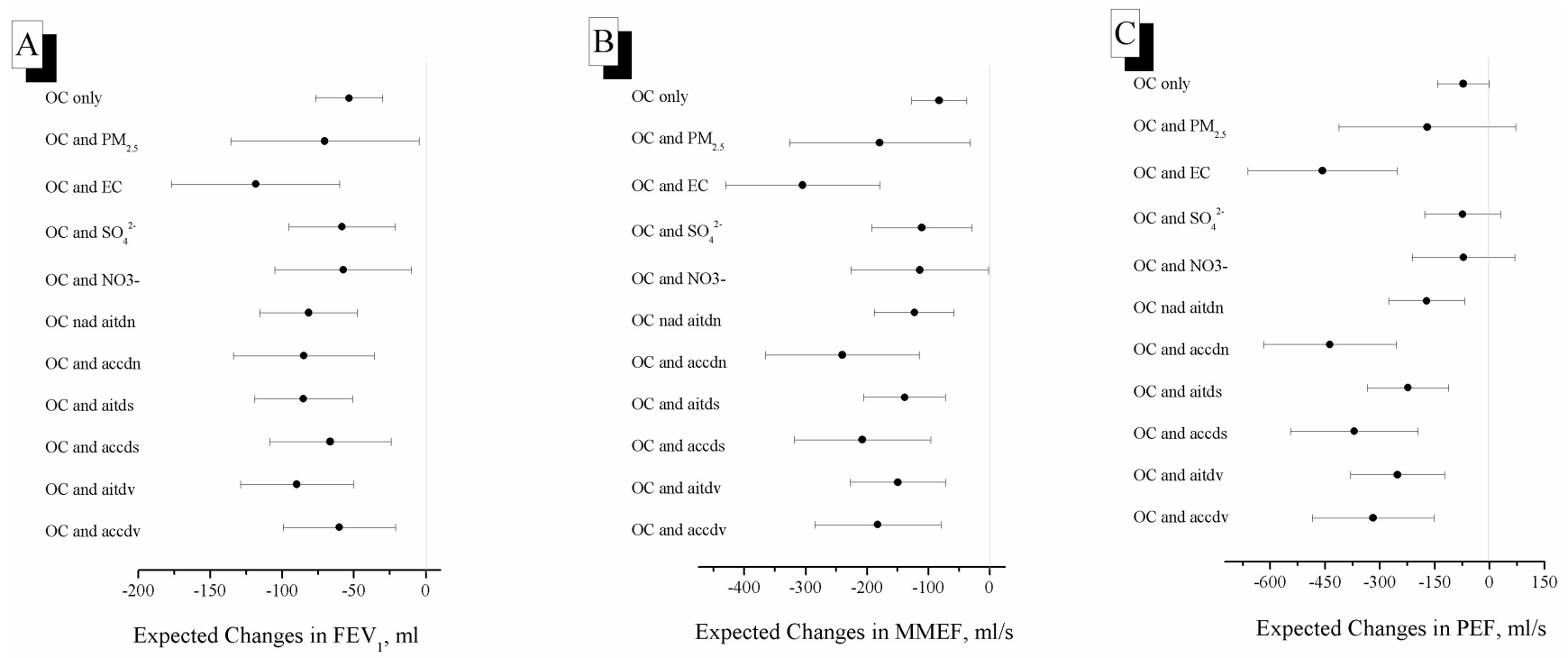

Figure S8. Changes in lung function from single pollutant models and two-pollutant models using 0-24h pollutant concentrations, Jiangmen, China, 2016. The correlation between the two pollutants was $\leq 0.8$. All models are adjusted for age, gender, BMI, ETS, temperature and RH, day-of-week and period. A for $\mathrm{FEV}_{1}$; B for MMEF; C for PEF.

\section{References}

1. Dong, H. B.; Zeng, L. M.; Hu, M.; Wu, Y. S.; Zhang, Y. H.; Slanina, J.; Zheng, M.; Wang, Z. F.; Jansen, R., Technical Note: The application of an improved gas and aerosol collector for ambient air pollutants in China. Atmos. Chem. Phys. 2012, 12 (21), 10519-10533.

2. Yue, D. L.; Hu, M.; Wang, Z. B.; Wen, M. T.; Guo, S.; Zhong, L. J.; Wiedensohler, A.; Zhang, Y. H., Comparison of particle number size distributions and new particle formation between the urban and rural sites in the PRD region, China. Atmospheric Environment 2013, 76, 181-188.

3. Willeke, K.; Baron, P. A., Aerosol Measurement Principles, Techniques, and Applications. . 1993.

4. Breiman, L., Random forests. Mach. Learn. 2001, 45 (1), 5-32.

5. Liaw, A.; Wiener, M., Classification and Regression by RandomForest. Forest 2001, 23.

6. Chen, X.; Liu, C. T.; Zhang, M.; Zhang, H., A forest-based approach to identifying gene and gene-gene interactions. Proc. Natl. Acad. Sci. U. S. A. 2007, 104 (49), 19199-19203.

7. Chen, X.; Ishwaran, H., Random forests for genomic data analysis. Genomics 2012, 99 (6), 323-329.

8. Liaw, A.; Wiener, M. RandomForest: Breiman and Cutler's random farests for classification and regression. https://cran.r-project.org/web/packages/randomForest/index.html. 